\title{
Expression pattern of the PRDX2, RAB1A, RAB1B, RAB5A and RAB25 genes in normal and cancer cervical tissues
}

\author{
ANDREJ NIKOSHKOV ${ }^{1}$, KRISTINA BROLIDEN ${ }^{2}$, SANAZ ATTARHA $^{1,3}$, VITALI SVIATOHA ${ }^{3}$, \\ ANN-CATHRIN HELLSTRÖM ${ }^{4}$, MIRIAM MINTS ${ }^{1}$ and SONIA ANDERSSON ${ }^{1}$ \\ ${ }^{1}$ Department of Women's and Children's Health, Division of Obstetrics and Gynecology, Karolinska Institute, \\ Karolinska University Hospital Solna, 17176 Stockholm; ${ }^{2}$ Department of Medicine Solna, \\ Unit of Infectious Diseases, Center for Molecular Medicine, Karolinska Institute, Karolinska University \\ Hospital, 17176 Stockholm; ${ }^{3}$ Department of Oncology-Pathology, Karolinska Institute, 17176 Stockholm; \\ ${ }^{4}$ Department of Gynecological Oncology, Radiumhemmet, Karolinska University Hospital, 17176 Stockholm, Sweden
}

Received July 11, 2014; Accepted August 28, 2014

DOI: 10.3892/ijo.2014.2724

\begin{abstract}
Cervical cancer is the second most prevalent malignancy among women worldwide, and additional objective diagnostic markers for this disease are needed. Given the link between cancer development and alternative splicing, we aimed to analyze the splicing patterns of the PRDX2, RAB1A, RAB1B, RAB5A and RAB25 genes, which are associated with different cancers, in normal cervical tissue, preinvasive cervical lesions and invasive cervical tumors, to identify new objective diagnostic markers. Biopsies of normal cervical tissue, preinvasive cervical lesions and invasive cervical tumors, were subjected to rapid amplification of cDNA 3' ends (3' RACE) RT-PCR. Resulting PCR products were analyzed on agarose gels, gel-purified and sequenced. Normal cervical tissue, preinvasive cervical lesions and invasive cervical tumors contained one PCR product corresponding to full-length PRDX2, RAB5A and RAB25 transcripts. All tissues contained two RAB1A-specific PCR products corresponding to the full-length transcript and one new alternatively spliced RAB1A transcript. Invasive cervical tumors contained one PCR product corresponding to the full-length RAB1B transcript, while all normal cervical tissue and preinvasive cervical lesions contained both the full-length RAB1B transcript and three new alternatively spliced RAB1B transcripts. Alternative splicing of the RAB1A transcript occurs in all cervical tissues, while alternative splicing of the
\end{abstract}

Correspondence to: Professor Sonia Andersson, Department of Women's and Children's Health, Division of Obstetrics and Gynecology, Karolinska Institute, Elevhemmet H2:00, Karolinska University Hospital Solna, 17176 Stockholm, Sweden

E-mail: sonia.andersson@karolinska.se

Key words: cervical cancer, alternative splicing, peroxiredoxin 2, RAB1B, member RAS oncogene family
RAB1B transcript occurs in normal cervical tissue and in preinvasive cervical lesions; not in invasive cervical tumors.

\section{Introduction}

Alternative splicing is a process in which either different combinations of exons or parts of exons are employed to generate new transcripts through the use of alternative-splicing sites. More than $90 \%$ of human intron-containing genes undergo alternative splicing, which allows a single transcript to encode a number of RNAs that produce various protein isoforms with different functions (1). A complex array of cis elements (the splicing code) embedded in pre-mRNAs, together with trans-acting factors that bind to these elements, are required for the precise process of pre-mRNA splicing and its regulation. The splicing code for the majority of introns comprises short sequences that include the $5^{\prime}$ and $3^{\prime}$ splice sites located at both intron-exon junctions and at the branch point. These sequences are recognized by the spliceosome, which is composed of five small nuclear ribonucleoproteins and $\sim 150$ proteins. In addition to the core cis elements in pre-mRNAs, other sequences in introns and exons, which are recognized by cellular trans-acting factors, are also critical to the determination of splicing outcomes (2).

In the last few years, a link has emerged between cancer development and deregulation of alternative splicing (3). Approximately $10 \%$ of inherited or somatic mutations are localized within sequences coding canonical splice sites (4), and these mutations have been implicated in cancer susceptibility and tumor progression. One such example is the tumor suppressor gene p53, the most commonly mutated gene in human cancers. Isoforms of the p53 gene are created through alternative splicing, and their tumor suppressor functions differ (5). 'Silent' mutations in the p53 gene have been predicted to affect splicing by creating splice sites in the middle of an exon (6). Recently, it has been reported that an attenuated form of familiar adenomatous polyposis is caused by the insertion of a single $\mathrm{T}$ between the second and third nucleotide of intron 4 of the APC gene, which leads to the 
skipping of exon 4, and a predicted expression of a truncated protein (7). Another good example of altered splicing in tumorigenesis occurs in the tumor suppressor gene BRCA1. Mutations in the BRCA1 gene are well-known markers of susceptibility to ovarian and breast cancer, the latter being the most common malignancy in women. One such mutation in the BRCA1 gene is an inherited nonsense mutation within exon 18 that disrupts the exonic splicing enhancer of the gene, provoking exon skipping (8).

Alterations in signaling pathways cause changes in the abundance, localization and activity of splicing regulators, which in turn can cause a general deregulation of splicing, and result in the occurrence of cancer-specific transcripts that are associated with tumorigenesis. Recent progress in molecular and cell biology has indicated that altered splicing profiles of critical genes may impact all aspects of cancer cell biology (9), resulting in the inactivation of tumor suppressor genes (6), or the gain of function of proteins implicated in cancer susceptibility and tumor progression $(9,10)$.

Human papillomavirus (HPV) infection is a necessary, but not sufficient factor in the development of cervical neoplasia, and persistent infection with high-risk HPV types, especially HPV16, is a significant risk factor in the development of precancerous lesions and squamous cell carcinoma (11-14). HPV-16, -18, -45, -31 and -33 are the most frequently identified high-risk HPV types in high-grade squamous intraepithelial lesions and squamous cell carcinoma (15), though HPV16 predominates (16). An association has also been suggested between high viral load and the persistence of HPV infection (17). Promising HPV-related markers of the risk of progression to cervical cancer include the integration status of high-risk HPV DNA in precancerous cervical lesions (18). HPV DNA integration into the host cell genome usually disrupts the E1 and E2 open reading frames, but leaves those of E6 and E7 intact $(19,20)$. The deletion of the E2 open reading frame due to HPV DNA integration leads to the disruption of E2 protein expression, and the upregulation of E6 and E7 protein transcription (21); continuous production of the oncogenic E6 and E7 proteins contribute to malignant progression.

It was recently demonstrated that level of the splicing activator ASF/SF2 (also known as arginine/serine-rich splicing factor 1) is modulated in the presence of HPV infection (22), as is the splicing silencer heterogeneous nuclear ribonucleoprotein A1 (hnRNP A1). The latter is a trans-acting factor that can modulate splice site selection (23), which may result in a change in the splicing pattern of a number of mRNAs expressed in HPV-infected cervical tissue. A previous proteomic analysis we preformed of vaginal and cervical cancer identified a number of tumor-specific markers (24). The biggest increase in cancer-related expression was observed for the prdx 2 protein, which was found to be upregulated in cervical cancer, and the Rablb protein (a member of the RAS oncogene family), which was found to be upregulated in both vaginal and cervical cancers. Based on these results, the present report further investigates the splicing patterns of the PRDX2 and RAB1B genes in normal cervical tissue, preinvasive cervical lesions and invasive cervical tumors. We also studied the expression patterns of other RAB gene members of the RAS oncogene family - RAB1A, RAB5A and RAB25, which are associated with different cancers.

\section{Materials and methods}

Between 2012 and 2013,28 women were enrolled in the present study: 10 healthy female volunteers (c1-c10), eight women with preinvasive cervical lesions (p1-p8), and 10 women with invasive cervical tumors (i1-i10). Each woman underwent a pelvic examination followed by biopsy collection at one of two departments in Stockholm, Sweden: the Division of Obstetrics and Gynecology, Karolinska University Hospital, or the Department of Gynecological Oncology, Radiumhemmet, Karolinska University Hospital. All biopsies were immediately flash frozen in liquid nitrogen and then stored at $-80^{\circ} \mathrm{C}$. Biopsies were grouped according to morphological diagnosis. Ethical approval was obtained from the Regional Ethics Review Board in Stockholm, and an informed consent form was signed by all participants prior to inclusion.

RNA preparations, cDNA synthesis. Approximately $30 \mathrm{mg}$ of biopsy tissue was homogenized in microtubes with disposable pestles driven by cordless motor (VWR, Radnor, PA, USA). Total RNA from homogenized biopsies was isolated using RNeasy kits (Qiagen, Hilden, Germany) according to the manufacturer's instructions. Total RNA (200 ng) was reverse transcribed with SuperScript III, and amplified with Platinum Taq DNA polymerase in $30 \mu \mathrm{l}$ of a one-step RT-PCR system (Invitrogen, Carlsbad, CA, USA) according to the manufacturer's instructions. The reverse transcription reaction was performed at $50^{\circ} \mathrm{C}$ to destroy mRNA secondary structures.

3'RACE RT-PCR. To detect multiple transcripts of the PRDX2, RAB1A, RAB1B, RAB5A and RAB25 genes, we applied the rapid amplification of cDNA $3^{\prime}$ ends (3' RACE) RT-PCR approach, which uses the natural polyA tail that exists at the $3^{\prime}$ end of most eukaryotic mRNAs for priming during reverse transcription. cDNAs were generated using an oligo-dT-adaptor GACTCGAGTCGACATCGATAG(T)19 primer that complements the polyA stretch and adds a special adaptor sequence to the 5' end of each cDNA. Further amplification of cDNAs in the one-step RT-PCR reaction was performed using the reverse TAILR, GACTCGAGTCGACATCGATAG primer targeting the adaptor sequence, and forward PRDX164F, CAGTC ATGGCCTCCGGTAA; RAB1A397F, CTGCAGTGACAT GTCCAGCAT; RAB1B16F, CCATGAACCCCGAATATGA CTA; RAB5A508F, CTGGAAGTTCATTGAAGAGTTGA; and 25RAB210F, CTCCATGCGGAGCCAAGAT primers, corresponding to sequences located in the 5' regions of the untranslated parts of the PRDX, RAB1A, RAB1B, RAB5A and RAB25 transcripts, respectively. As these forward primers are located in front of the initiation ATG codons, PCR products corresponding to both the PRDX or RAB transcripts included the entire translated region in each case. The resulting PCR products were gel-purified using QIAquick gel extraction kits (Qiagen), and then further amplified and sequenced by BigDye Terminator kits (Applied Biosystems, Foster City, CA, USA) using the internal reverse primers PRDX864R, GGT CCCATACTGTGGAGTT; RAB1A2522R, CCATGTATTT CAATTGCCTGTT (or RAB1A1957R, CTACCTCTACCAC AGATGCATT); RAB1B1701R, GACTTGCTTTCTTGCAG GAAGCA (or RAB1B1551R, TCCCTGGTGGGCTCCAG AGA); RAB5A2493R, CCAACCTGAGCACCTCAATATA 

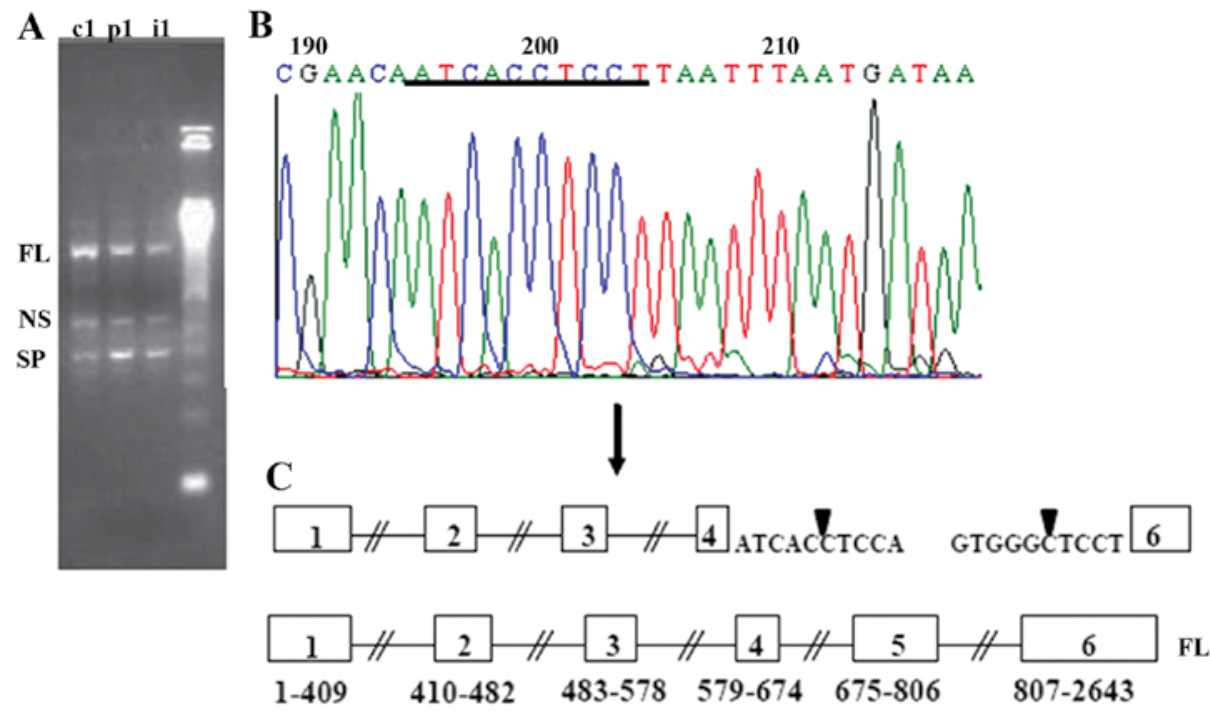

MSSMNPEYDYLFKLLLIGDSGIGKSCLLLRFADDTYTESYISTIGIDFKIR $\Delta S p$ TIELDGKTI KLQIWDTAGQERFRTITS(79)LI

Figure 1. RAB1A amplification products. Structure of RAB1A mRNAs and protein variants. (A) Amplification products from RAB1A transcripts expressed in normal cervical tissue (c1), preinvasive cervical lesions (p1) and invasive cervical tumors (i1). (B) Chromatogram of junction site in spliced RAB1A mRNA. (C) Structure of full-length and spliced human RAB1A mRNAs and of the spliced Rabla protein. FL, PCR product corresponding to the full-length mRNA in cervical tissues; NS, 550 bp non-specific fragment; SP, 400 bp PCR product corresponding to alternatively spliced mRNAs. Junction site in spliced RAB1A mRNA is underlined on chromatogram or marked by an arrow. Boxes with numbers correspond to exons. The first base of the ATG initiation codon which is located in exon 1 is arbitrarily designated +1 . Triangles indicate the excised part of RAB1A mRNA. $\triangle \mathrm{Sp}$, predicted sequences of RAB1B protein isoforms. Number in brackets corresponds to the position of the last amino acid of the RAB1B protein within the new peptide.

(or RAB5A2057R, CCTATTTACAGTACAGCTGAAGAT); and 25RAB1077R, GGACAGATAAAAGAGGTATTTGTG which correspond to the sequences located within untranslated 3' regions of the PRDX, RAB1A, RAB1B, RAB5A and RAB25 genes, respectively. RAB1A, RAB1B, RAB5A and RAB25 internal reverse primers were also used in 40 cycles of nested PCR for further analyses of non-specific (NS) DNA generated from the first round of PCR and separated on agarose gels.

\section{Results}

Total RNAs from normal cervical tissue, preinvasive cervical lesions and invasive cervical tumors, were screened for the presence of PRDX, RAB1A, RAB1B, RAB5A and RAB25 transcripts by a sensitive RT-PCR approach. The primers used in the PCR reaction spanned the entire coding region of all five genes, and each case included the initiation ATG codon and the polyA tail. All PRDX amplification products from normal cervical tissue, preinvasive cervical lesions and invasive cervical tumors consisted of a single PCR product generated from the full-length transcripts (data not shown).

All RAB1A amplification products from all samples of normal cervical tissue, preinvasive cervical lesions and invasive cervical tumors consisted of a long PCR product and two shorter 400 and $550 \mathrm{bp}$ bands (Fig. 1A). All these products, were cut from the gel, and the DNA was extracted and amplified by nested PCR employing the RAB116F primer (the same forward primer used in the first round of PCR) and the RAB1A2522R primer, which targets the sequence located within the 3 ' untranslated region of the transcript. This round of amplification generated only one shorter ( 400 bp)
RAB1A-specific band, which was also extracted from the gel and sequenced using the RAB1A397F forward and RAB1A2522R or RABA1957R reverse primers. Sequence analysis revealed that a long PCR product (FL) was generated from the full-length transcripts while $400 \mathrm{bp}$ band correspond to a new spliced transcripts (SP) where non-classical splicing sites were used to excise the RAB1A sequence between exons 4 and 6 (Fig. 1B and C).

To eliminate possibility of missing RAB1A transcripts which did not amplify sufficiently due to some cDNA/PCR reaction bias, and were thus undetectable in gel analyses of first-round PCR product, we also analyzed by nested PCR DNA extracted from all samples in the areas between FL and NS bands, NS and SP bands and SP band and 250 bp but did not find any new RAB1A-specific PCR fragments.

RAB1B amplification products from invasive cervical tumors consisted of a single PCR product generated from the full-length transcripts of this gene (Fig. 2C), while amplification products from all normal cervical tissue and preinvasive cervical lesions consisted of a shorter PCR product of $\sim 600 \mathrm{bp}$, together with some visible less abundant amplification products in the range of 400-500 bp (Fig. 2A and B). To further analyze full-length and shorter products, they were cut from the gel, and the DNA was extracted and amplified by a nested PCR reaction employing the RAB116F primer (the same forward primer as used in the first round of PCR) and the RAB1B1701R reverse primer targeting the sequence located within the 3' untranslated region of the transcript. This round of amplification generated three new RAB1B-specific PCR products which were sequenced using the RAB116F forward and RAB1701R and RAB1551R reverse primers. We also 
A

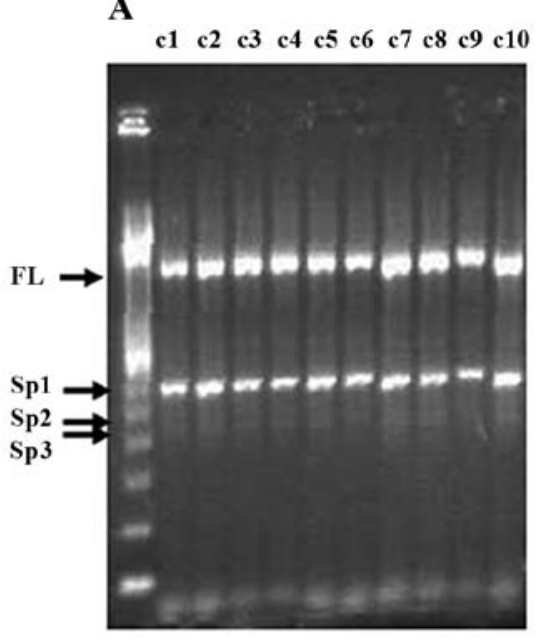

B
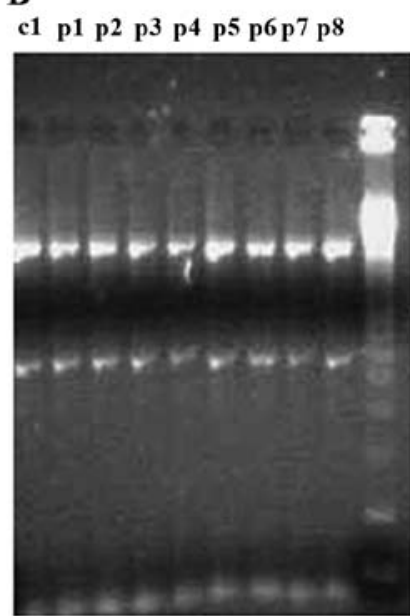

C

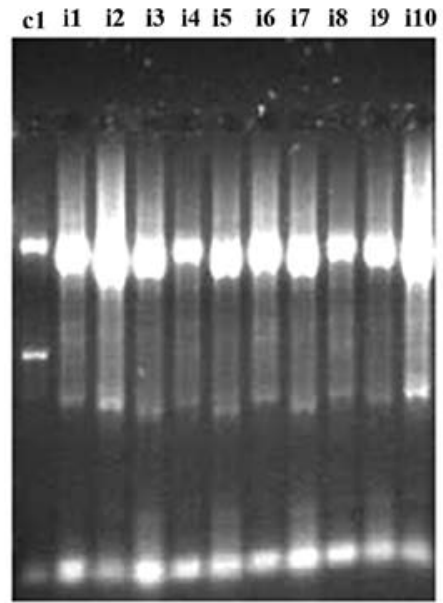

Figure 2. RAB1B amplification products. (A) Amplification products from RAB1B transcripts expressed in normal cervical tissue (c1-c10). (B) Amplification products from RAB1B transcripts expressed in preinvasive cervical lesions (p1-p8). The first lane was loaded with sample c1. (C) Amplification products from RAB1B transcripts expressed in invasive cervical tumors (i1-i10). The first lane was loaded with sample c1. PCR product c1 ( $2 \mu 1)$ and PCR product i1-i10 ( $8 \mu 1)$ from standard RT-PCR reactions, which included $200 \mathrm{ng}$ of total RNA, were loaded on the gel. FL, 1,630 bp PCR product corresponding to the full-length mRNA in normal cervical tissue; Sp1, Sp2, Sp3 are 600, 484 and 432 bp PCR products corresponding to alternatively spliced mRNAs. Sp2 and Sp3 are weakly visible in the first PCR reaction and were further amplified by nested PCR.

further amplified first round PCR material from normal and preinvasive tissues in the range 250-400 and 500-1400 bp (i.e., excluding full-length and 400-500 bp product) but did not detect any shorter RAB1B-specific PCR products.

RAB1B amplification products from invasive cervical tumors consisted of a single PCR product generated from the full-length transcripts of this gene. As no visible bands corresponding to the shorter RAB1B-specific products (found in normal tissues or preinvasive lesions) were detected in invasive tumors after analyzing of $2 \mu \mathrm{l}$ (equal for all samples in all groups) of standard RT-PCR reactions (data not shown), we analyzed larger $(8 \mu \mathrm{l})$ volumes of the RT-PCR reactions from invasive tumors (Fig. 2C). However, no visible bands corresponding to new shorter Rablb-specific products were observed. DNA from the regions of the gel corresponding approximately to the range $250-1400 \mathrm{bp}$ (i.e., including the visible product at $\sim 270 \mathrm{bp}$ (Fig. 2C), but excluding the prominent full-length product) and also from the region containing the product corresponding to full-length cDNA, were extracted from the gel and subjected to nested PCR. However, this yielded no RAB1B-specific products, suggesting that all this material represents NS amplification products from the first round of PCR reactions, in which cDNAs of invasive tumor material were used as templates.

An interesting feature of the RAB1B alternative splicing pattern was that the pattern of expression was similar for all individuals of the same group, reflecting the existence of common mechanisms for the generation of new transcripts.

The RT-PCR experiments were designed so that the RNAs of the same amount were treated in the same way from the same master mix in three independent experiments. Thus, these results serve as good controls for RNA integrity and confirm that the new RAB1B forms detected in the study are not artifacts. Sequence analysis revealed that all of the shorter RAB1B-specific PCR products Sp1, Sp2 and Sp3 (Fig. 3) correspond to three new transcripts with sizes of 600,484 and 422 bp, respectively. In the Sp1 and Sp3 transcripts, classical splicing sites are used to excise the RAB1B sequence between exons 4 (Sp1) or 3 (Sp3), and exon 6, while the Sp2 transcript is generated due to splice sites comprised of non-classical splicing signals (Fig. 3).

All RAB5A and RAB25 amplification products from normal cervical tissue, preinvasive cervical lesions and invasive cervical tumors consisted of a single PCR product corresponding to the full-length transcripts of RAB5A and RAB25 (data not shown). We extracted all DNA in the area between $250 \mathrm{bp}$ and the full-length band from all samples and amplified DNA extracts by nested PCR employing the same forward primers used in the first round of PCR, and reverse RAB5A- and RAB25-specific primers targeting the sequence located within the 3 ' untranslated regions of the transcripts. We did not detect any new RAB5A- or RAB25-specific PCR products.

\section{Discussion}

In this study we investigated transcription of PRDX2, RAB1A, RAB1B, RAB5A and RAB25 genes in normal cervical tissue, preinvasive cervical lesions and invasive cervical tumors. PRDX2 and RAB1B gene expression was found to be associated with cervical cancer in our proteomics study (24). The RAB1A gene is structurally and functionally very similar to the RAB1B gene, and is associated with the occurrence of tongue and prostate cancers $(25,26)$. The RAB5A gene is also similar to the $\mathrm{RAB} 1 \mathrm{~B}$ gene; it is upregulated in cervical cancer and expresses a protein that is a key regulator of intracellular vesicle traffic from the plasma membrane to early endosomes. Silencing of RAB5A expression in human cervical carcinoma in HeLa and SiHa cell lines decreased cell motility and invasiveness (27). RAB25 gene expression is upregulated in ovarian and breast cancers, and the level of Rab25 protein overexpression is associated with more aggressive cancer forms (28).

We found that only the closely related RAB1A and RAB1B genes could express alternatively spliced RNAs in cervical 


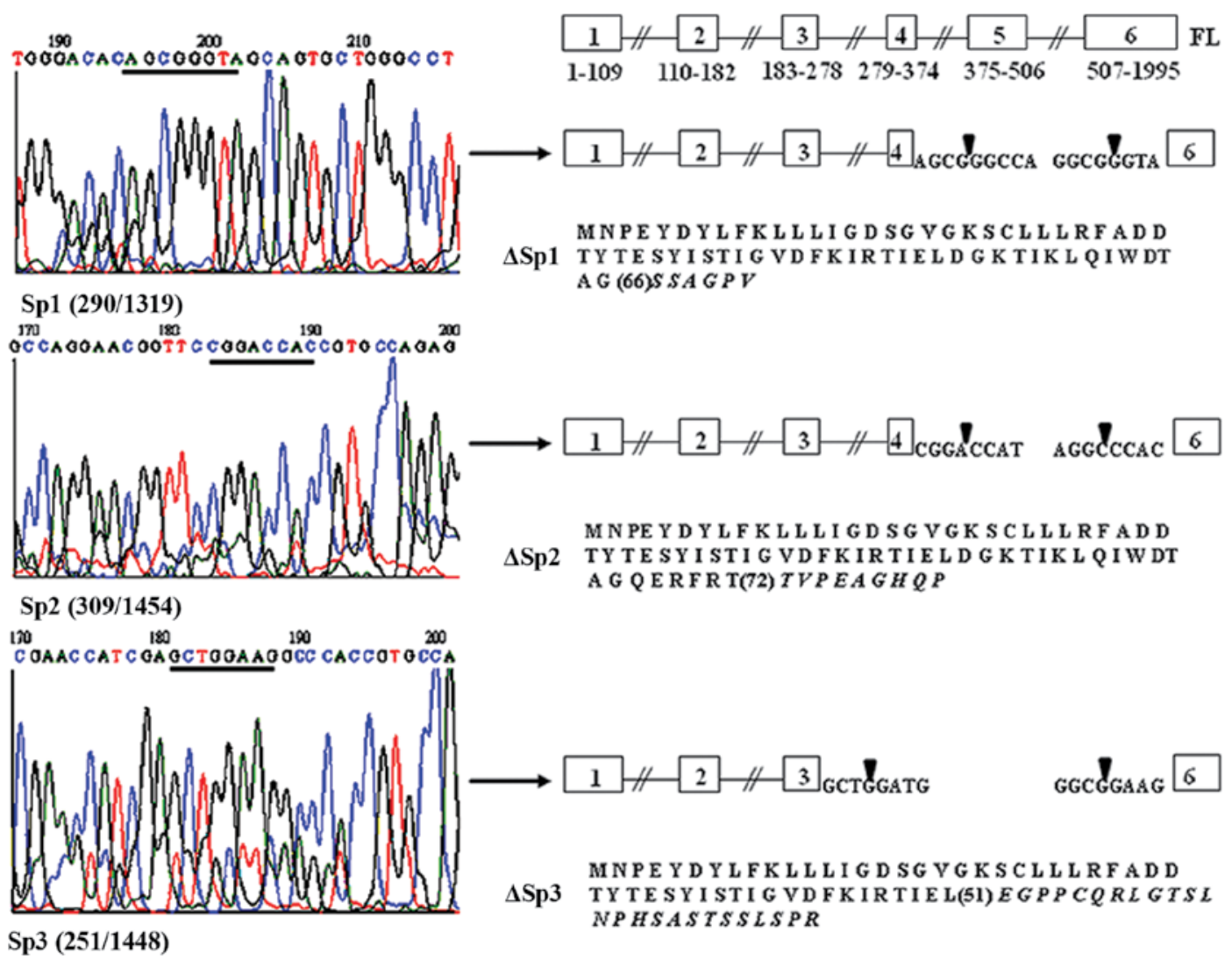

Figure 3. Structure of RAB1B mRNAs and protein variants. Numbers show the positions of the boundaries of new introns. Junction sites in spliced RAB1B mRNAs are underlined on chromatograms or marked by an arrow. FL, structure of full-length human RAB1B mRNA. Boxes with numbers correspond to exons. The first base of the ATG initiation codon which is located in exon 1 is arbitrarily designated +1 . Triangles indicate the excised part of RAB1B mRNA. $\Delta \mathrm{Sp} 1, \Delta \mathrm{Sp} 2, \Delta \mathrm{Sp} 3$ : Predicted sequences of RAB1B protein isoforms. Numbers in brackets correspond to the position of the last amino acid of the RAB1B protein within the new peptide. Italics correspond to a new peptide sequence.

tissue. No spliced forms of the RAB1A or RAB1B genes have been described thus far, making the findings described in this study novel. We also demonstrate that new RAB1B mRNA transcripts, which are expressed in normal cervical tissue and preinvasive cervical lesions, are downregulated in invasive cervical tumors.

Both RAB1A and RAB1B genes consist of five introns and six exons (Figs. 1 and 2), and belong to a group of small Ras-related GTP-binding proteins encoded by RAB genes, and participate in the regulation of vesicular transport from the endoplasmic reticulum to the Golgi in mammalian cells. Many of the $>60$ different human Rab proteins that have been identified have been localized to discrete subcellular compartments (29).

All new RAB1A and RAB1B transcripts found in this study contain an initiation translation codon ATG, and therefore should be capable of being translated into proteins. An interesting feature of such proteins $(\Delta \mathrm{Sp}, \Delta \mathrm{Sp} 1, \Delta \mathrm{Sp} 2$ and $\Delta \mathrm{Sp} 3)$ is that all of them are the products of short reading frames (1-79 aa for $\Delta \mathrm{Sp}, 1-66$ aa for $\Delta \mathrm{Sp} 1,1-72$ aa for $\Delta \mathrm{Sp} 2$ and 1-51 aa for $\Delta \mathrm{Sp} 3$ ) in which the $\mathrm{N}$-terminus of the RAB1B gene is followed by very short, unspecific amino acid sequences (Figs. 1 and 2). The Rabla and Rablb protein sequences are very similar and contain several functional domains. A detailed domain/function description for the $\mathrm{Rabla} / \mathrm{b}$ proteins is not yet available, but it is still possible to predict that the new proteins contain one or two GTP-binding sites, for Rablb protein at positions $15-22$ and $63-67$, respectively (http://www.uniprot.org/uniprot/Q9H0U4). All of the other known domains of the Rablb protein: the Swich 2, a determinant of nucleotide-dependent functions aa 64-84, the CAAX box which directs post-translational modifications, and the hypervariable region targeting Rablb to specific membranes (both located near the - $\mathrm{COOH}$ terminus of the protein), are missing from the new Rablb (and most probably from Rab1a) proteins. Both Ras and several Rab proteins are known to be implicated in the development of different cancers $(25-28,30)$. Variations in Ras protein expression have tumorigenic potential, since membrane traffic is of key importance in cancer biology due to its influence on cell polarity in the metastatic transformation of tumor cells (29). Ras/Rab proteins are involved in malignant progression, as they are key components of the signal transduction pathways triggered by different mitogens (30). Because Rab proteins can interact with their molecular targets in the GTP bound conformation, it could be hypothesized that tissue-specific alternative splicing and translation of both Rabla/b and likely other Rab proteins as well, could generate peptides that only retain GTP-binding sites; thus the multitude of such peptides effectively translated from the short reading frames could influence the intracellular concentrations of GTP, which regulates tumorigenesis and other cellular functions. The disappearance of the alternative transcripts of the RAB1B gene in invasive cervical cancer could be related to known molecular mechanisms of HPV 
tumorigenesis. In cervical cancer, one of the key events of malignant transformation is HPV DNA integration into the host chromosome. This is followed by the sustained expression of the viral late genes E6 and E7, whilst other portions of the viral DNA are deleted or undergo transcription disturbance (31). Such events could reduce cellular levels of the splicing activator ASF/SF2 (activated by the HPV E2 protein) in tumors, while the splicing silencer hnRNP A1 is upregulated after HPV infection (23). Thus the splicing of a number of genes, including RAB1B, which may be dependent on the ASF/SF2: hnRNP A1 ratio, could be inhibited.

We used nested PCR to amplify all RAB1A, RAB1B, RAB5A and RAB25 first-round PCR DNA products extracted from all agarose gel areas which did not contain visible bands, but did not find any gene-specific products. This eliminated possibility of missing potential transcripts which did not amplify sufficiently due to some cDNA/PCR reaction bias, and were thus undetectable in gel analyses of first-round PCR products.

In conclusion, these results suggest that multiple, alternatively spliced RAB1A/B transcripts are generated in human cervical tissue and may be translated into short proteins that are capable of GTP binding. The expression of RAB1B transcripts is inhibited in invasive cervical tumors, probably due to a shift in the HPV-regulated ratio between splicing activators and splicing inhibitors. A study of other members of the Ras/Rab family in the same types of tissue as those in this study would be of great interest to determine whether the silencing of alternative splicing identified in this study has a more general impact on cervical cancer development. Analysis of RAB1B may also help to identify women who are at risk of developing cervical cancer. Indeed, the definition of an objective molecular marker to identify these women is of extreme importance, as cytomorphological identification has proven difficult, and the percentage of HPV positivity in young women is high. Therefore, we plan to conduct both retrospective studies using archival liquid-based cytology samples, and prospective randomized studies using routinely collected slides.

\section{Acknowledgements}

We wish to thank Trudy Perdrix-Thoma for editing assistance and English language review.

\section{References}

1. Black DL: Mechanisms of alternative pre-messenger RNA splicing. Annu Rev Biochem 72: 291-336, 2003.

2. Singh RK and Cooper TA: Pre-mRNA splicing in disease and therapeutics. Trends Mol Med 18: 472-482, 2012.

3. Pal S, Gupta R and Davuluri RV: Alternative transcription and alternative splicing in cancer. Pharmacol Ther 136: 283-294, 2012.

4. Garcia-Blanco MA, Baraniak AP and Lasda EL: Alternative splicing in disease and therapy. Nat Biotechnol 22: 535-546, 2004.

5. Mills AA: p53: link to the past, bridge to the future. Genes Dev 19: 2091-2099, 2005.

6. Lamolle G, Marin M and Alvarez-Valin F: Silent mutations in the gene encoding the p53 protein are preferentially located in conserved amino acid positions and splicing enhancers. Mutat Res 600: 102-112, 2006.
7. Neklason DW, Solomon CH, Dalton AL, Kuwada SK and Burt RW: Intron 4 mutation in APC gene results in splice defect and attenuated FAP phenotype. Fam Cancer 3: 35-40, 2004.

8. Mazoyer S, Puget N, Perrin-Vidoz L, Lynch HT, SerovaSinilnikova OM and Lenoir GM: A BRCA1 nonsense mutation causes exon skipping. Am J Hum Genet 62: 713-715, 1998.

9. Ghigna C, Valacca $\mathrm{C}$ and Biamonti G: Alternative splicing and tumor progression. Curr Genomics 9: 556-570, 2008.

10. David CJ and Manley JL: Alternative pre-mRNA splicing regulation in cancer: pathways and programs unhinged. Genes Dev 24: 2343-2364, 2010.

11. Bosch FX and Muñoz N: The viral etiology of cervical cancer. Virus Res 89: 183-190, 2002.

12. Walboomers JM, Jacobs MV, Manos MM, et al: Human papillomavirus is a necessary cause of invasive cervical cancer worldwide. J Pathol 189: 12-19, 1999.

13. zur Hausen H: Papillomaviruses and cancer: from basic studies to clinical application. Nat Rev Cancer 2: 342-350, 2002.

14. Muñoz N, Bosch FX, de Sanjosé S, et al: Epidemiologic classification of human papillomavirus types associated with cervical cancer. N Engl J Med 348: 518-527, 2003.

15. Muñoz N: Human papillomavirus and cancer: the epidemiological evidence. J Clin Virol 19: 1-5, 2000.

16. Clifford GM, Smith JS, Plummer M, Muñoz N and Franceschi S: Human papillomavirus types in invasive cervical cancer worldwide: a meta-analysis. Br J Cancer 88: 63-73, 2003.

17. Sundström K, Ploner A, Dahlström LA, et al: Prospective study of HPV16 viral load and risk of in situ and invasive squamous cervical cancer. Cancer Epidemiol Biomarkers Prev 22: 150-158, 2013.

18. Lazo PA: Papillomavirus integration: prognostic marker in cervical cancer? Am J Obstet Gynecol 176: 1121-1122, 1997.

19. Kalantari M, Karlsen F, Kristensen G, Holm R, Hagmar B and Johansson B: Disruption of the E1 and E2 reading frames of HPV 16 in cervical carcinoma is associated with poor prognosis. Int J Gynecol Pathol 17: 146-153, 1998.

20. Kalantari M, Blennow E, Hagmar B and Johansson B: Physical state of HPV16 and chromosomal mapping of the integrated form in cervical carcinomas. Diagn Mol Pathol 10: 46-54, 2001.

21. Romanczuk H and Howley PM: Disruption of either the E1 or the E2 regulatory gene of human papillomavirus type 16 increases viral immortalization capacity. Proc Natl Acad Sci USA 89: 3159-3163, 1992.

22. McPhillips MG, Veerapraditsin T, Cumming SA, et al: SF2/ASF binds the human papillomavirus type 16 late RNA control element and is regulated during differentiation of virus-infected epithelial cells. J Virol 78: 10598-10605, 2004.

23. Cheunim T, Zhang J, Milligan SG, McPhillips MG and Graham SV: The alternative splicing factor hnRNP A1 is up-regulated during virus-infected epithelial cell differentiation and binds the human papillomavirus type 16 late regulatory element. Virus Res 131: 189-198, 2008.

24. Hellman K, Alaiya AA, Becker S, et al: Differential tissue-specific protein markers of vaginal carcinoma. Br J Cancer 100: 1303-1314, 2009.

25. Shimada K, Uzawa K, Kato M, et al: Aberrant expression of RAB1A in human tongue cancer. Br J Cancer 92: 1915-1921, 2005.

26. Sun T, Wang X, He HH, et al: MiR-221 promotes the development of androgen independence in prostate cancer cells via downregulation of HECTD2 and RAB1A. Oncogene 33: 2790-2800, 2014.

27. Liu SS, Chen XM, Zheng HX, Shi SL and Li Y: Knockdown of Rab5a expression decreases cancer cell motility and invasion through integrin-mediated signaling pathway. J Biomed Sci 18: $58,2011$.

28. Cheng KW, Lahad JP, Kuo WL, et al: The RAB25 small GTPase determines aggressiveness of ovarian and breast cancers. Nat Med 10: 1251-1256, 2004

29. Hutagalung AH and Novick PJ: Role of Rab GTPases in membrane traffic and cell physiology. Physiol Rev 91: 119-149, 2011.

30. Hernández-Alcoceba R, del Peso L and Lacal JC: The Ras family of GTPases in cancer cell invasion. Cell Mol Life Sci 57: 65-76, 2000.

31. Münger K, Baldwin A, Edwards KM, et al: Mechanisms of human papillomavirus-induced oncogenesis. J Virol 78: 11451-11460, 2004. 